\title{
NEUROCIENCIA Y TEORÍA POLÍTICA FEMINISTA. LA INESTABILIDAD SEXO-GÉNERO-SEXUALIDAD A TRAVÉS DE LA OBRA DE PAUL B. PRECIADO'
}

\author{
MARIA MEDINA-VICENT \\ Universitat Jaume I
}

\begin{abstract}
RESUMEN: Hoy en día las noticias que vienen acompañadas del prefijo «neuro-» son acogidas con gran entusiasmo por el público general así como por el académico. La autoridad que se otorga a los resultados de los experimentos neurocientíficos y su exitosa divulgación a través de los medios de masas, sirven para que la lectura neurocientífica del dimorfismo sexual se haya extendido rápidamente, abriendo viejos debates feministas. Desde una posición feminista, nos vemos en la obligación de aproximarnos críticamente al neurogénero y sus derivaciones sexistas conocidas como neurosexismo. Esta empresa se realiza con el objeto de identificar los riesgos que dicho discurso entraña para la lucha política feminista. Para conseguir nuestro objetivo nos basaremos en el análisis de las obras seminales del feminismo, así como en la propuesta postfeminista de Paul B. Preciado.
\end{abstract}

PALABRAS CLAVE: neurociencias, neurogénero, neurosexismo, neurofeminisno, ética feminista.

\section{Neuroscience and Feminist Political Theory. The Instability Sex-Gender-Sexuality Through the Work of Paul B. Preciado}

\begin{abstract}
Today news about neuroscience is received with great enthusiasm by the general public as well as the academic field. The authority awarded to the results of neuroscientific experiments, linked to its successful dissemination through the mass media, serves to extend the sexual dimorphism argument, opening old feminist debates. Consequently, from a feminist position, we ought to critically approach the neurogenderings and the neurosexism. This aim is performed in order to identify the risks that such discourse entails for feminist political struggle. In order to achieve our objective, we rely on the analysis of the seminal works of feminism, as well as the post-feminist proposal of Paul B. Preciado.
\end{abstract}

KEY WORDS: neuroscience, neurogenderings, neurosexism, neurofeminism, feminist ethics.

\section{INTRODUCCIÓN}

El discurso neurocientífico se ha convertido en una de las bases culturales de las sociedades occidentales contemporáneas. En su intención por desentrañar el funcionamiento del cerebro humano, dicha disciplina hace uso de diferentes técnicas (resonancia magnética, electroencefalograma, magnetoencefalograma, etc.) que supuestamente permiten «ver» el cerebro en su estructura y funcionamiento. Sin embargo, la operación desarrollada a través de dichas máquinas, así como la lectura que los/as neurocientíficos/as realizan de los

1 Este trabajo se inscribe en el Proyecto de Investigación Científica y Desarrollo Tecnológico FI2013-47136-C2-2-P, financiado por el Ministerio de Economía y Competitividad del Gobierno de España. 
resultados, no se encuentran en el ámbito de la objetividad. Más bien al contrario, tanto el diseño como la ejecución y posterior interpretación de los experimentos neurocientíficos se realizan culturalmente, con todo lo que esto supone (prejuicios, sesgos de género, de raza, etc.). Y es que, debemos reconocer que ni la ciencia es un ámbito neutral, ni lo son el diseño y el uso de las máquinas con las que se elaboran los estudios. Por tanto, la pretensión normativa derivada de los mismos debe ser revisada desde un punto de vista ético (Salles, 2013).

En este sentido, debemos tener presente el impacto de dichos resultados en la construcción de las identidades sociales y de género, sin dejar de advertir los riesgos que esto supone para la fundamentación de una sociedad verdaderamente igualitaria. Partiendo de esta idea, nuestra tarea será aproximarnos críticamente a los discursos que las neurociencias efectúan sobre las diferencias estructurales entre los cerebros de hombres y mujeres, desde las obras seminales del feminismo, hasta la propuesta postfeminista de Paul B. Preciado. La principal razón que nos lleva a emprender este estudio es la incidencia que dicho discurso puede tener en la perpetuación de las desigualdades de género, en la medida en que renueva los tradicionales argumentos biologicistas sin ofrecer una perspectiva crítica de los mismos.

De este modo, construiremos una crítica al neurosexismo desde un ensamblaje feminista lo suficientemente sólido como para mostrar que las bases filosóficas del feminismo desarrolladas desde el siglo XvIII hasta el postfeminismo actual, aportan las fórmulas necesarias para desmontar el carácter biologicista de algunas de las teorías neurocientíficas. Pondremos especial énfasis en la propuesta postfeminista de Paul B. Preciado, quien se erige como sujeto de la experimentación, mostrando en su propio cuerpo la fragilidad de las fronteras que tradicionalmente han separado a un género de otro y a un sexo de otro. Y es que, al fin y al cabo, en el caso de que se pueda comprobar empíricamente que los cerebros de mujeres y hombres cuentan con diferentes estructuras o que funcionan de modos distintos, nuestra obligación es preguntarnos si realmente dicha diferenciación (históricamente argumentada desde posiciones naturalistas) debe influir en la constitución de una sociedad democrática e igualitaria. Avanzando nuestra posición, entendemos que dichas comprobaciones empíricas tendrían sus ventajas como avances en el conocimiento del cerebro humano, pero que resultarían estériles en nuestro camino hacia la justicia social.

La estructura que sigue el presente artículo es la siguiente. En primer lugar, se realizará una aproximación al concepto de neurosexismo para entrever sus riesgos para la práctica feminista, y cómo dichos riesgos han fomentado la aparición del neurofeminismo. En segundo lugar, intentaremos demostrar que las bases morales, políticas y filosóficas que ha desarrollado la teoría feminista desde el siglo XVIII son fuente suficiente para desmontar el esencialismo que impregna al neurosexismo. Por último, nos centraremos en la filosofía postfeminista de Paul B. Preciado, ya que su propuesta nos permite desmantelar el neurosexismo desde una posición diferente, mostrando que no solamente el género es un constructo cultural, sino que también lo son los sexos y los cuerpos, lo que hace posible transitar de unos constructos a otros, de unos cuerpos a otros. 
2. El POTENCIAL ÉTICO DEL NEUROFEMINISMO FRENTE A LOS RIESGOS MORALES DEL NEUROSEXISMO

El ámbito neurocientífico es productor de conocimiento y creador de discurso social, ahora también, sobre la cuestión de género. Cuando empezaron a proliferar los estudios neurocientíficos sobre el género, existió una primera intención de unir los intereses de las teorías feministas con las neurociencias, dando lugar al concepto neurogenderings o neurogénero (Kraus, 2012). Sin embargo, los resultados de dichos experimentos y sobre todo, la divulgación mediática de las teorías sobre el dimorfismo sexual desde posiciones que no se abren al debate (Rodríguez-Sierra, 2016), pronto se tornaron en contra de la lucha feminista. Teniendo en cuenta esta realidad, no es de extrañar que las posturas feministas acusen a parte de las neurociencias, de realizar una lectura positivista de sus resultados, y de divulgarlos como si se tratase de verdades absolutas.

Así, las críticas feministas han abordado diferentes cuestiones, desde el ámbito teórico, a las carencias de los diseños de las investigaciones neurocientíficas, hasta la necesidad de tornar transparentes los procesos que llevan a dicha disciplina a establecer conclusiones cerradas sobre las diferencias cerebrales entre mujeres y hombres (Longino, 1993). Esta ardua empresa que se ha llevado a cabo desde el neurofeminismo se inició con la obra de Anne Fausto-Sterling titulada Myths of Gender: Biological Theories about Women and Men (1992). Con la misma intención se produjo el surgimiento del concepto Neurosexism (Fine, 2010), que permite señalar el acriticismo que impregna a parte de la práctica investigadora neurocientífica, así como a la difusión de sus resultados.

En vistas a denunciar el impacto que tienen las producciones pseudocientíficas de las neurociencias en la vida diaria de los individuos, en su condición de mujeres y hombres, el concepto Neurosexism nos otorga el potencial crítico que necesitamos para emprender nuestro trabajo. Así pues, teniendo en cuenta que el concepto de neurosexismo nos permitiría identificar el problema, el concepto neurofeminismo nos permitiría crear el espacio y la posición crítica desde la que reflexionar sobre dicho problema. De este modo, la labor central del neurofeminismo sería la de poner en jaque el supuesto carácter absoluto de los descubrimientos neurocientíficos, mostrando que la importancia de la cultura en la conformación del cerebro resulta vital para comprender la edificación de las identidades de género, que no serían constructos cerrados.

En resumen, las derivas reduccionistas del discurso neurocientífico sobre el género, han hecho que los feminismos se replanteen los lazos que le empezaban a unir con las neurociencias, para mostrar su cara más crítica. Este apartado nos ha servido para comprobar que es un error considerar que han sido las neurociencias las primeras en tratar la cuestión de género. Al contrario, la teoría crítica feminista lleva siglos aportando reflexiones valiosas sobre las relaciones de género, que ponen en un segundo plano el carácter determinante de lo biológico, otorgándole valor a lo cultural. En el siguiente apartado nos 
centraremos en mostrar que aunque realmente exista un dimorfismo sexual entre los cerebros de mujeres y hombres, cuán poco condicionante es para el desarrollo de sus vidas en igualdad. De este modo, pondremos de relieve la operación reduccionista que realiza parte de las neurociencias, al pasar por encima de toda una corriente filosófica y de lucha política: los feminismos.

\section{LAS OBRAS SEMINALES DEL FEMINISMO FRENTE A LOS «NUEVOS» ARGUMENTOS NEUROSEXISTAS}

Se ha señalado que las neurociencias aplicadas al estudio del género se presentan ante nosotros/as como verdades absolutas acerca de la diferenciación entre mujeres y hombres. Sin embargo, desde una perspectiva crítica podemos preguntarnos: ¿Qué novedades nos aportan las neurociencias con respecto al género? ¿Son sus argumentos tan diferentes de los esgrimidos históricamente por el patriarcado? En el caso de que sus argumentos sean ciertos, ¿son realmente determinantes para el desarrollo del feminismo como vía de búsqueda de la justicia social? Partiendo de dichas cuestiones, nuestra hipótesis de partida en este punto es que los descubrimientos de las neurociencias con respecto al dimorfismo sexual no resultan tan diferentes de los argumentos naturalistas históricamente rebatidos por el feminismo, al tiempo que no son condicionantes para los avances conseguidos por dicho movimiento político. Para determinar si las neurociencias aportan algo nuevo sobre las diferencias entre hombres y mujeres debemos saber lo que siglos antes ha dicho el feminismo para rebatir dichas diferencias construidas en clave de desigualdad.

En primer lugar, debemos reconocer que las neurociencias han realizado un uso sesgado y poco profundo del concepto género. Como se ha señalado anteriormente, mientras que los/as teóricos/as feministas se aproximan al género como un constructo complejo de distintos niveles, algunos/as neurocientíficos/ as reducen los comportamientos de mujeres y hombres a sus características cerebrales (Jordan-Young y Rumiati, 2012: 105). Mediante esta visión reduccionista olvidan que dicho concepto nace con la intención de señalar críticamente la desigualdad entre mujeres y hombres construida a través de la articulación de diferentes dimensiones de lo social: económica, política e incluso tecnológica. Así pues, aunque el término "género» puede resultar a veces controvertido, lo vamos a entender aquí como la «forma de referirse a la organización social de las relaciones entre sexos» (Scott, 1996: 266).

Reducir el género a una serie de características de la estructura cerebral, dejando de lado su valor como categoría que denuncia las desigualdades sociales entre mujeres y hombres, le resta todo su potencial transformador. Al fin y al cabo, toda disciplina que muestre interés en su estudio, debe comprometerse con un análisis multidimensional del fenómeno de la desigualdad, que pretenda explicar la opresión de las mujeres, para comprender en mayor medida los procesos de exclusión sexual que aún perviven en nuestras sociedades. Desde 
mi punto de vista, el estudio del género por parte de las neurociencias muestra un escaso compromiso con un análisis que dialogue con otras disciplinas, perpetuando una visión de los cerebros de mujeres y hombres de corte determinista. Se trata de una reformulación de la dualidad de género y la «justificación de las diferencias». Es decir, el tipo de enraizamiento de ciertas marcas en el cuerpo a través de las ciencias del cerebro.

En segundo lugar, a lo largo del desarrollo histórico de la lucha feminista, los argumentos patriarcales naturalistas ahora retomados por las neurociencias han sido debatidos por diferentes autoras y corrientes. Por esta razón, realizaremos un pequeño recorrido por las obras seminales del feminismo, que nos mostrarán que muchas mujeres lucharon hace tiempo por enfrentar los argumentos reduccionistas que pretendían fundamentar en una supuesta naturaleza, la desigualdad entre hombres y mujeres.

La Declaración de los Derechos de la Mujer y la Ciudadana (1789, Olympe de Gouges) nos sirve como punto de partida para comprender la evolución del feminismo a lo largo de los siglos, así como para apreciar el recorrido filosófico, político y social que tiene en comparación a las recientes neurociencias. En estas primeras posiciones del feminismo, a pesar de poder identificar cierto sesgo esencialista, queda clara la necesidad de cambiar las estructuras sociales, políticas y económicas para fomentar la igualdad de género. Es decir, a pesar de que no se puede obviar en estas primeras posiciones una afirmación de la diferencia biológica entre mujeres y hombres como una ventaja para la superioridad moral de las primeras - lo vemos en afirmaciones como «el sexo superior tanto en belleza como en coraje, como en los sufrimientos maternos» (hablando de las mujeres) (Preámbulo de la Declaración) — esto no impide que las feministas se aperciban de que para transformar la realidad, hay que realizar acciones materiales. Por ejemplo, en la afirmación «la ignorancia, el olvido o el desprecio de los derechos de la mujer son las únicas causas de los males públicos y de la corrupción de los gobiernos» (Preámbulo de la Declaración), se ve claramente cómo se asocia la consecución del bienestar de las mujeres a la lucha política. Al mismo tiempo, se puede deducir que uno de los mayores deseos de estas mujeres es poder participar en la toma de decisiones, en la administración de la cosa pública (Artículo 14). Así pues, vemos que ya en los textos fundacionales del movimiento feminista se articula la dimensión material y estructural de la desigualdad de género, mostrándonos la poca relevancia que las diferencias físicas, biológicas y corporales — muchas veces reivindicadas por las propias feministas en esta época- deben tener en el fundamento de una sociedad democrática.

Por tanto, aunque en estos primeros estadios de la lucha feminista se reconozca la existencia de una diferenciación natural entre mujeres y hombres, no se la concibe como un elemento determinante para condicionar la vida de las mujeres. Más bien al contrario, vemos claramente el compromiso con la reconstrucción de una sociedad igualitaria, moldeando la naturaleza a través de la cultura. En este sentido, Mary Wollstonecraft pone en juego un discurso muy potente sobre la importancia de la educación de las mujeres en su obra 
Vindicación de los derechos de la mujer (1792). La autora «defiende el derecho de las mujeres a la educación para alcanzar la independencia económica que las situaría en un plano de igualdad frente a los hombres y critica la tiranía del placer y la belleza» (Alberola, 2012: 44). Frente al argumento naturalista referido a la debilidad del sexo femenino, esgrimido por las instituciones del siglo XVIII, Wollstonecraft señala que la libertad de las mujeres no puede estar condicionada por un rasgo tan frágil y fugaz como la belleza, debe basarse en algo más fuerte, como la educación que nos otorga herramientas de lucha.

Tomando esta idea esgrimida hace poco más de dos siglos, venimos a reivindicar de nuevo que la igualdad y la libertad de las mujeres no pueden estar condicionadas a ningún atributo físico, o en este caso neuronal, ya que dichos aspectos no resultan condicionantes en el fomento de una sociedad igualitaria y verdaderamente justa. En resumen, los feminismos de la primera ola (Siglos XVIII y XIX) «pretenderán que la filosofía, como corpus teórico, ha de admitir que la capacidad de razonar es neutral respecto del sexo, aunque ello no implique que no haya diferencias racionales que puedan ser explicadas por diferencias corporales» (Reverter, 2003: 37). Es decir, que la capacidad de razonamiento tanto de mujeres como de hombres les pertenece en tanto seres humanos, y no en base a sus diferencias biológicas, que no deben ser consideradas ante las estructuras de cualquier Estado Social que se precie.

Por otro lado, encontramos la Declaración de Seneca Falls o Declaración de Sentimientos (1848), cuya convención fue impulsada por Elizabeth Cady Stanton y Lucretia Mott en el siglo xIx. Ambas mujeres, «tomando como modelo la Declaración de la Independencia (1776) de Thomas Jefferson, redactaron la Declaración de Sentimientos en la que se incluyen unos dieciocho motivos de quejas de las mujeres» (Alberola, 2012: 45). De su lectura se desprende de nuevo la importancia de la educación como herramienta clave para luchar contra los argumentos naturalistas, una educación que ha sido negada a las mujeres: «He has denied her the facilities for obtaining a thorough education - all colleges being closed against her» (Declaration of Sentiments en Coy, 1993: 72). Además, en la declaración se señala que el carácter dócil de las mujeres de la época poco tenía que ver con su "naturaleza», sino más bien con la situación de sometimiento a la que eran condenadas: «He has endeavored, in every way that he could to destroy her confidence in her own powers, to lessen her self-respect, and to make her willing to lead a dependent and abject life» (Declaration of Sentiments en Coy, 1993: 72).

Así pues, la segunda ola del feminismo (Segunda mitad del siglo xix y primer tercio del xx) denunció que aquella supuesta naturaleza que había relegado a las mujeres a un segundo plano en todos los ámbitos sociales, distaba mucho de ser una realidad dada e inamovible. En vistas a luchar contra los argumentos que las esclavizaban a una supuesta naturaleza primera, tomaron medidas de actuación y reclamación, para ocupar el espacio público que les había sido negado.

Avanzando en el tiempo, nos situamos frente al ensayo feminista más importante del siglo xx: El segundo sexo (1949) de Simone de Beauvoir, que defiende 
la construcción cultural de las diferencias entre mujeres y hombres. En este escrito, De Beauvoir «desenmascara los elementos ideológicos — los prejuicios, los tópicos, los puntos de inflexión de la ideología masculina- que perpetúan y producen la situación de opresión en la que viven las mujeres» (Alberola, 2012: 49). Uno de sus aspectos más interesantes reside en la reflexión que la autora realiza sobre el papel determinante de la cultura y los condicionantes sociales en la determinación de nuestros roles sociales. En este sentido, para dicha autora una de las razones centrales sobre las que se sustenta la dominación femenina, sería el menosprecio cultural de la capacidad reproductiva de las mujeres, por debajo de la capacidad productiva de los hombres. Esta idea resulta clave, ya que vemos cómo las capacidades y responsabilidades que les han sido adjudicadas a las mujeres en base a una supuesta naturaleza primigenia, son articuladas desde, por y para la cultura. Esto quiere decir que nuestra posición social viene determinada por un amplio complejo de factores, y que por tanto: «No se nace mujer, se llega a serlo». Se pone de relieve que no existen las esencias masculina o femenina. Por esta razón, educar en valores éticos es una de las herramientas más poderosas que tiene el feminismo ante sí, ya que permite transformar las desigualdades.

Como vemos, en el desarrollo de la tercera ola del feminismo (Segunda mitad del siglo xx y comienzos del xxi), y siguiendo las enseñanzas de Simone De Beauvoir, se entiende que la posición social de mujeres y hombres viene determinada por un conjunto complejo de condicionantes culturales, políticos, tecnológicos, etc. donde la propia biología tiene poco de determinante. Por tanto, que desde las neurociencias se caiga en un neurosexismo que pretenda encontrar la «esencia femenina» en el cerebro, supone volver a viejos argumentos y dar un paso atrás en los logros de la teoría feminista.

Pero profundicemos más en este aspecto clave del feminismo, y en su poder para denunciar las construcciones sociales que legitiman la desigualdad de género y relegan a las mujeres a una posición de inferioridad social, a través de otra obra seminal: La mistica de la feminidad (Friedan, 1963). A través de esta obra la estadounidense Betty Friedan «retrató con gran precisión las dificultades que experimentaba la mujer norteamericana de clase media» (Alberola, 2012: 49, identificando «el malestar sin nombre» de las estadounidenses. Muchas mujeres acudían al psicoanalista en un intento por mejorar su situación mental, sin embargo, como señala la misma Friedan: «(...) la extraña novedad del malestar es que no se puede entender desde el prisma de los eternos problemas materiales del hombre: la pobreza, la enfermedad, el hambre, el frío» (Friedan, 2009: 62). Quizá si las neurociencias hubieran estado lo suficientemente desarrolladas en dicha época, habrían intentado dar una explicación neuronal a dicho malestar. Sin embargo, ¿qué sentido tendría realizar dicha operación? Realmente, una explicación neuronal para dicho malestar habría sido poco productiva, ya que como la propia Friedan indica en su obra, la solución al problema pasa por la toma de conciencia de las mujeres de que los modelos de feminidad que les son impuestos son eso, modelos de conducta que en muchos casos no les van a aportar la felicidad. Por esta razón, la autora reivindica que 
el ser mujer está compuesto de muchas facetas, de muchas identidades que se superponen y convergen (madre, trabajadora, amante, intelectual, etc.). El único modo de articular de forma sana dichas identidades es si las estructuras sociales nos lo permiten y esto va más allá de las estructuras cerebrales que tengamos, atañe a cuestiones de poder.

Desde nuestro punto de vista, el neurosexismo puede estar empezando a reconstruir un discurso patriarcal que pretende naturalizar la desigualdad entre mujeres y hombres a través de sus diferencias cerebrales. Y es que, no debemos olvidar que la producción de conocimiento científico ha sido históricamente un dominio masculino, construido por y para dicho grupo. En este sentido, no resulta descabellado pensar que los estereotipos y los prejuicios sexistas también impregnan la práctica neurocientífica (Bluhm, 2013), y en la medida en que esto es así, debemos plantearnos la recepción de sus experimentos de forma crítica y feminista (Fisher, 2015).

En resumen, hemos podido observar cómo desde el siglo xVIII, han sido diversas las autoras que han esgrimido sus argumentos por la consecución de la igualdad dentro de la corriente feminista. Aunque existen corrientes de corte esencialista, comprendemos que el eje vertebral de la lucha feminista se centra en reconocer el carácter construido de los roles sociales y del papel de la categoría de género para señalar las relaciones de poder que subyacen a la desigualdad entre mujeres y hombres. Así pues, hablemos de quien hablemos, ya sea Olympe de Gouges, Mary Wollstonecraft, Elizabeth Cady Stanton, Simone de Beauvoir o Betty Friedan; una parte de la lucha feminista nos revela que el verdadero potencial de transformación, o al menos el más inmediato, no se refiere tanto a los componentes físicos, biológicos o neurales como a los culturales y materiales. Desde diferentes argumentos y posiciones, el feminismo nos muestra que lo que verdaderamente fundamenta las diferencias entre hombres y mujeres, no se encuentra ni en su cerebro ni en su naturaleza, si es que algo así existe; sino en los papeles sociales que se les asignan en función de su sexo y las estructuras sociales de poder que los legitiman.

\section{PostFeminismo: FÁRMACOS, BISTURÍS Y LA PLASTICIDAD DE LOS CUERPOS}

En nuestro intento por revelar el escaso poder de fundamentación que los estudios neurocientíficos tienen a la hora de abordar la cuestión de género, intentaremos subrayar la plasticidad de los cuerpos como herramienta para la distorsión de las categorías irrefutables de hombre y mujer, que el neurosexismo se afana por defender. Para realizar dicha tarea nos centraremos en la propuesta filosófica de Paul B. Preciado, que nos permitirá mostrar que la fisicidad de los cuerpos, así como de la biología e incluso del cerebro, no son condicionantes finales en la definición de los géneros, los sexos ni las sexualidades. Ya que debido a la plasticidad de los cuerpos, la cuestión biológica también puede transformarse. 
Habíamos avanzado anteriormente una de las cuestiones centrales del debate entre las neurociencias y los feminismos: el uso que las primeras realizan de la categoría género (Browne, 2016), así como su confusión de los términos sexo y género, cuestión abordada por la profesora Sonia Reverter-Bañón en su artículo "Reflexión crítica frente al neurosexismo» (2016), dentro de este mismo número. Dicho constructo teórico cuenta con un gran recorrido, e incluso dentro de las propias filas feministas prevalece un intenso debate en torno a su definición. De este modo, la tendencia a su reificación por parte de las neurociencias, empobrece notablemente el debate feminista, ya que simplifica su complejidad semántica. Por tanto, mientras que en los estudios de género dicha categoría responde a un complejo entramado de factores culturales, sociales, políticos e incluso económicos que condicionan las relaciones humanas y la conformación de las instituciones sociales; en el campo de las neurociencias se convierte a menudo en una dicotomía simplista, es decir, varones con un género y hembras con otro, con sus comportamientos, actitudes, aptitudes y habilidades correspondientes, haciendo que «masculinidad y feminidad se conviertan en cosas predefinidas y sistemas cerrados con un aval científico» (Pallarés-Domínguez, 2011: 21).

En este sentido, el trabajo de Preciado desarrollado centralmente en sus obras Manifiesto contra-sexual (2002) y Testo yonki (2008), nos permite concebir la materialidad de los cuerpos en la que se basan las afirmaciones neurocientíficas anteriores, como un proceso en constante construcción. Así pues, nuestra hipótesis central de argumentación se centra en la idea de que si con los avances farmacéuticos y quirúrgicos con los que contamos hoy en día, somos capaces de transformar nuestros cuerpos pasando de un sexo a otro, e incluso construyendo cuerpos no normativos, qué importancia puede tener que hombres y mujeres tengan diferentes estructuras cerebrales, si al fin y al cabo, nuestros cuerpos son plásticos y por tanto, susceptibles de transformación. Y no solamente susceptibles de transformación a través del bisturí, sino también a través del uso de fármacos, hormonas, y otras sustancias. Por ejemplo, el uso de la píldora anticonceptiva permite a las mujeres no quedarse embarazadas. Así, mediante la ingesta de una pastilla se está reconstruyendo su cuerpo y su identidad de género, quitándoles aquello que históricamente las ha caracterizado de forma "natural» como mujeres: su capacidad reproductiva y de albergar vida dentro de su ser.

El trabajo de Preciado se inscribe en el postfeminismo, caracterizado por la descentralización del sujeto político de dicho movimiento, pasando de una noción cerrada de mujer a una incorporación de los grupos sociales hasta ahora excluidos en las reclamaciones feministas: lesbianas, gays, transexuales, transgénero, etc. En el año 2002 Preciado publicó Manifiesto contra-sexual, obra clave en el desarrollo de la teoría y el movimiento queer. Partiremos de esta obra para tratar el concepto de "contra-sexualidad», que contiene la intención de superar una noción natural de sexo y sexualidad. Dicho concepto nos permitirá llegar al meollo de la cuestión, esto es, la noción de los cuerpos como constructos plásticos, artificiales y modificables. 
La contra-sexualidad es entre otras cosas «una teoría del cuerpo que se sitúa fuera de las oposiciones hombre/mujer, masculino/femenino, heterosexualidad/ homosexualidad» (Preciado, 2002: 19). Con la voluntad de des-naturalizar y des-mitificar las nociones tradicionales de sexo, sexualidad y género, la contrasexualidad «estudia los instrumentos y los aparatos sexuales y, por lo tanto, las relaciones de sexo y de género que se establecen entre el cuerpo y la máquina» (Preciado, 2002: 21). De este modo, se conceptualiza el sexo como tecnología, y se conjuga con la idea de "prótesis de género", que resulta de vital importancia para repensar los procesos de asignación de sexo, género y sexualidad en la sociedad occidental. A partir de dicha definición, se pueden extraer dos ideas principales. En primer lugar, que las prácticas heterosexuales «naturales» son el fruto de un contrato artificial, cuya artificialidad intenta hacer patente Preciado mediante la propuesta de otro contrato, el contra-sexual. En segundo lugar, la propuesta contrasexual nos remite a la idea de que no solamente el género es construido cultural y socialmente, sino que también los cuerpos biológicos lo son. Desde esta última premisa edificaremos nuestro segundo argumento contra el neurosexismo.

Recopilemos pues nuestro primer argumento contra el neurosexismo derivado de la conjunción entre neurociencias y estudios de género. Hemos visto que a lo largo de la historia del feminismo, los principales argumentos se centraban en el carácter cultural y construido de los roles sociales de género, que asignaban diferentes valores a las biologías femeninas y masculinas, asignándoles una posición de inferioridad a los primeros cuerpos. De esta manera, era la cultura la principal encargada de crear las diferencias entre mujeres y hombres, el cuerpo se llegaba a entender como una especie de lugar inicial sobre el que efectuar dichas construcciones. Teniendo en cuenta esta idea, entendíamos que en el caso de que realmente existan diferencias considerables ente los cerebros de mujeres y hombres, la cultura y la educación pueden acabar con dichas diferencias. Ahora bien, nuestro segundo argumento tomará en cuenta el papel de los cuerpos a la hora de subvertir también esas supuestas diferencias cerebrales que las neurociencias defienden con sus experimentos. A través de la teoría de Preciado, veremos que no solamente la cultura puede transformar los roles de género, sino que también los cuerpos pueden transformarse para acabar con esa supuesta naturaleza biológica que nos ata a un género $\mathrm{u}$ a otro.

En primer lugar, expondremos la cuestión referida a la heterosexualidad supuestamente natural. En este sentido, Preciado toma los trabajos de Judith Butler para reconocer desde la idea de performatividad (Butler, 2007), los mecanismos de normalización de género que son puestos en práctica desde las estructuras estatales, educativas, médicas, etc., en vistas a fundamentar la heteronormatividad como algo natural. Desde este punto de vista, se entiende que el discurso heteronormativo se inscribe en los cuerpos a través de diferentes tecnologías del poder (Foucault, 2008). De este modo, Butler concibe las identidades de género como construcciones que se realizan a través de los discursos institucionalizados sobre esas mismas identidades. Es decir, son construcciones culturales realizadas a través del lenguaje, las instituciones y los discursos 
(donde podríamos incluir el neurocientífico) que se dan a través de un proceso disciplinario en el que la sanción ejerce un papel central. Así, toda aquella conducta, apariencia, etc. que se escape de la norma será sancionada en un intento por reconducirla hacia lo establecido, legitimando así su existencia como desviación; y fundamentando las identidades de género supuestamente naturales.

Esta idea guarda una estrecha relación con el concepto foucaultiano de biopolítica, mediante el que Michel Foucault «observa el conjunto de fenómenos que tuvieron lugar entre los siglos XVII y XVIII, cuando el Estado comienza a ejercer su soberanía sobre los cuerpos y despliega lo que puede denominarse una «tecnología de la seguridad biológica aplicada sobre el cuerpo» (Aguilera, 2010: 33). Dicho concepto nos remite a las estrategias de poder que tienen por objeto ejercer un control total sobre la vida de las personas y sus cuerpos en la sociedad moderna. En esta línea, el poder político para producir verdades se inscribe sobre los cuerpos a través de la biopolítica, que se encuentra en todos los ámbitos sociales. Por tanto, el concepto de performatividad empleado por Butler, sirve para discernir el poder que tienen los discursos sociales en la configuración de los cuerpos en función de unas identidades de género concretas. En este mismo sentido, entendemos que el discurso neurocientífico del dimorfismo sexual ejerce su papel como mecanismo para la producción de las identidades de género tradicionales y del sistema heteronormativo.

A través de los enunciados de género se legitima y recrea una verdad política, entendida como la reafirmación de las identidades de género dicotómicas y de la sexualidad heterosexual. Dichos actos performativos reproducen una convención social. La teoría de Butler conduce a la redefinición de la noción de género, que afirma que la identidad de género no sería algo sustancial, sino el efecto performativo de la invocación de una serie de convenciones de feminidad y masculinidad. En esta línea, la materialidad que sostiene las identidades de género es también construida como arquitectura política, ya que está fragmentada en dicotomías: permitido/no permitido, activo/pasivo, masculino/ femenino, etc. Además de ser performativo como diría Butler, Preciado señala que «el género es ante todo prostético, es decir, no se da sino en la materialidad de los cuerpos. Es puramente construido y al mismo tiempo enteramente orgánico. Escapa a las falsas dicotomías metafísicas entre el cuerpo y el alma, la forma y la materia» (Preciado, 2002: 25). Se reconoce así no solamente el carácter constructivista de los géneros, sino el hecho de que la materialidad corpórea sobre la que se edifican también es construida, no es una verdad primigenia sobre la que se inscriben los significados culturales.

Para llegar a comprender estas afirmaciones, debemos introducirnos en la noción del sexo como tecnología. Según esta perspectiva, no existen cuerpos terminados, no existen cuerpos finitos. El sexo es tecnología que se reinventa a través de las prótesis. Así pues, Preciado advierte de que uno de los mayores errores del feminismo ha sido tornar esenciales los cuerpos como si se tratasen de un punto cero desde el que se va a construir después el género, y esto es algo que comparte con Butler: 
La división sexo/género y la categoría de sexo en sí parecen dar por sentada una generalización de «el cuerpo» que existe antes de la obtención de su significación sexuada. Con frecuencia, este "cuerpo» parece ser un medio pasivo que es significado por la inscripción de una fuente cultural percibida como «externa» respecto de él. No obstante, cualquier teoría del cuerpo culturalmente construido debería poner en duda «el cuerpo» por ser un constructo de generalidad dudosa cuando se entiende como pasivo y anterior al discurso (Butler, 2007: 254).

En términos generales, la teoría feminista no renuncia en ningún momento a ese cuerpo biológico aparentemente imperturbable. Se parte «de un presupuesto moderno: la creencia según la cual el cuerpo entraña un grado cero o verdad última, una materia biológica (...) dada» (Preciado, 2002: 126), al igual que lo hacen parte de las neurociencias. Sin embargo, los avances en la transformación de los órganos sexuales y los cuerpos mediante intervenciones quirúrgicas, muestran que no es solamente el género lo que se construye cultural y normativamente hablando, sino el cuerpo orgánico el que es construido y modificado a golpe de bisturí y fármacos. Por tanto, apoyándonos en su visión, encontramos el fundamento filosófico necesario para defender que la supuesta diferencia entre los cerebros de mujeres y hombres resulta en gran medida, irrelevante, ya que no solamente el cuerpo no debe ser un condicionante importante en permitir la igualdad de mujeres y hombres, como se diría desde los feminismos tradicionales, sino porque desde la visión postfeminista, el cuerpo es moldeable y puede ser cambiado a través de las prótesis que transforman la materialidad del cuerpo, y desde las herramientas y sustancias que nos presta la industria farmacéutica.

Para ilustrar la artificialidad de los géneros y los sexos, Preciado reflexiona sobre la asignación de sexo a los bebés intersexuales y los casos de personas transexuales. Al nacer da comienzo el proceso de asignación sexual que será institucionalizado a lo largo de la vida del sujeto a través de instituciones como la familia, la escuela y el Estado. En su trabajo, nos hace reflexionar sobre la escasa cientificidad de los procesos en que un bebé es reconocido como niño o niña, ya que la elección es ante todo visual. En los casos de intersexualidad, donde los órganos no se corresponden con la norma, ya sea por tamaño, forma u otras cuestiones, la determinación de si dicho bebé será una mujer o un hombre, resulta igualmente gratuita. Un ejemplo revelador que expone Preciado, es el caso de bebés cuyo pene es más pequeño de lo «normal». En muchas ocasiones se decide convertir dicho pene en una vagina para ahorrar supuestos trastornos futuros. Como vemos, la identidad sexual se decide de forma visual, y es transformada tecnológicamente. Así, los bebés intersexuales ponen en funcionamiento dos tecnologías diferenciadas, una genética esencialista y otra quirúrgica constructivista (Preciado, 2002: 113).

En base a la creencia de una dualidad cero de cuerpos (masculino y femenino), todo aquello que no entre dentro de dicha pareja, será intervenido tecnológicamente para construirse como tal. Asignar a estos bebés un sexo y un género contiene valor prescriptivo, no descriptivo. Crear un órgano sexual 
determinado, poner un nombre propio... son enunciados prescriptivos que intervendrán en la formación de esa persona como mujer u hombre heterosexual. Por tanto, se esclarece un hecho y es que no existe una naturaleza sexual o genérica preexistente, sino que la misma acción de nombrarla inscribe sobre los cuerpos unos significados concretos. Además, dicha «naturaleza» se puede construir directamente a través de fármacos y bisturís. En relación, la lectura de las neurociencias sobre el dimorfismo sexual se configuraría como un enunciado prescriptivo que pugna por la desigualdad entre dos categorías opuestas: mujeres y hombres. Por tanto, las aportaciones que proceden del ámbito neurocientífico en referencia a estas cuestiones, resultarían poco relevantes en cuanto a la definición de un género u otro, ya que desde el postfeminismo entendemos que dichas nociones dicotómicas están más que obsoletas.

En sus esfuerzos por revelar la fragilidad de las nociones cerradas de género, sexo y sexualidad, Preciado experimentó en su propio cuerpo un proceso de masculinización. Los cambios físicos que la administración de testosterona tuvieron sobre su cuerpo, catalogado como femenino en el momento de su nacimiento, fueron registrados en su obra Testo yonki (2008). Sus análisis y experimentaciones son una muestra de que la facticidad corpórea no puede tomarse como elemento final de la determinación del género, sexo o sexualidad. En definitiva, lo que nos muestra la obra de Preciado es la posibilidad de transitar diferentes géneros, sexos y sexualidades a lo largo de la vida. De transformar las nociones dicotómicas y abrir paso a nuevas posibilidades identitarias (Preciado en Curia, 2016).

A través de las reflexiones de Preciado y Butler hemos podido observar que la regulación de los cuerpos bajo un sistema heteronormativo se nutre de los aparatos médicos, educativos, mediáticos, etc. para configurar aquello considerado natural. Entendemos que dicho discurso también se nutre de las aportaciones de las neurociencias, que mediante la fundamentación científica de las diferencias cerebrales entre mujeres y hombres, contribuye a la construcción cultural de la supuesta naturaleza ontológica de los sexos, los cuerpos, las sexualidades y los géneros. Y es que si tenemos en cuenta, como nos muestra el trabajo de Preciado, el poder de la tecnología, la industria farmacéutica y la médica para transformar los cuerpos y las identidades, podemos deducir que, aun en el caso de que existan diferencias cerebrales entre mujeres y hombres, éstas no son solamente irrelevantes desde un punto de vista postfeminista, sino que son susceptibles de modificación mediante fármacos, o intervenciones. Algo que al fin y al cabo, pone de relieve la futilidad del cuerpo como determinante de nuestros comportamientos, al contrario de lo que sostiene el neurosexismo.

\section{Conclusión}

El ámbito de las neurociencias se encuentra atravesado por los estereotipos de género. Obviar que este espacio de producción de conocimiento también 
reproduce las desigualdades de género, es obviar una realidad patente. A grandes rasgos, hemos podido ver que en gran parte de los estudios neurocientíficos centrados en el género, se realiza una lectura sesgada de los resultados (Reverter-Bañón, 2016), pasando por alto la importancia de los factores culturales en la conformación de los roles de género y las contribuciones de la teoría feminista a los mismos. Esta realidad sumada a la gran aceptación de sus postulados entre el público, nos obligan a reflexionar críticamente acerca de su poder en la perpetuación de la desigualdad. Así pues, el auge de las neurociencias «ha dejado patente una gran pertinencia normativa y metodológica, sin duda alguna derivada de la ciencia experimental propia del cientificismo positivista que la acompaña desde hace dos siglos» (Pallarés-Domínguez, 2013: 85). Dicha herencia positivista hace que las neurociencias se resistan a establecer un diálogo crítico con otras disciplinas, mostrando su generalizado carácter monológico. En el caso de los estudios de género, esto supone un riesgo mayor, ya que las neurociencias pueden acabar por realizar una fundamentación esencialista de las diferencias entre mujeres y hombres.

Atendiendo a esta realidad y al poder cada vez mayor de estos discursos neurosexistas en el seno de lo social, resulta imprescindible abordar las neurociencias desde la ética (Cortina, 2011; García-Marzá y Feenstra, 2013), y desde un punto de vista feminista como el que hemos intentado desarrollar aquí. En este sentido, las aportaciones históricas de algunas de las obras clave del feminismo nos muestran que en el caso de que existan diferencias considerables entre las biologías de mujeres y hombres, eso no debería importar para la consideración social igualitaria de ambos grupos. Es decir, por el lado del feminismo tradicional, podemos ver que en el camino a la igualdad de género y a la justicia social, las diferencias biológicas (existentes o no) se convierten en un asunto de poca importancia, ya que lo verdaderamente importante radicaría en los derechos, las estructuras sociales y la cultura. Mientras que en las teorías postfeministas, se nos permite entrever que es posible desestabilizar las dicotomías y las nociones de género tradicionales no solamente a través de la cultura, sino también a través de la transformación de los cuerpos. Así, revelan que los cuerpos son moldeables y/o plásticos y que por tanto, no suponen un condicionante inamovible por lo que respecta a las construcciones identitarias de género, ya que su fisicidad está sujeta a transformaciones. Y es que si tenemos en cuenta, como nos muestra el trabajo de Preciado, el poder de la tecnología, la industria farmacéutica y la médica para transformar los cuerpos y las identidades, podemos deducir que, aun en el caso de que existan diferencias cerebrales entre mujeres y hombres, éstas no son solamente irrisorias en un plano físico, sino que son susceptibles de modificación mediante fármacos o intervenciones. Por tanto, no deben constituirse como condicionantes fundamentales de los roles de género dicotómicos.

En conclusión, frente a los argumentos neurosexistas que se desprenden de muchos de los experimentos neurocientíficos sobre el género, podemos señalar que las supuestas diferencias entre las estructuras cerebrales de mujeres y hombres no son determinantes a la hora de conformar su identidad. Y es que, 
dicha identidad de género se puede transformar tanto a través de la cultura como a través de la intervención en la fisicidad de los cuerpos.

\section{Bibliografía}

Aguilera, R. E., «Biopolítica, poder y sujeto en Michel Foucault» en: Universitas, Revista de Filosofía, Derecho y Política, Nº11, 2010, 27-42.

Alberola, N., «Una definición polémica: la identidad femenina», en: Torrent, R. y ReVERTER, S. (Eds.), Variaciones sobre el género, Editorial ACEN, Castelló de la Plana 2012, 43-54.

Bluhm, R., "New Research, Old Problems: Methodological and Ethical Issues in fMRI Research Examining Sex/Gender Differences in Emotion Processing» en: Neuroethics, Vol.6, N², 2013, 319-330.

BRowne, T. K., "Why parents should not be told the sex of their fetus» en: Journal of Medical Ethics, Published Online First, 2016, 1-6.

Butler, J., El género en disputa. El feminismo y la subversión de la identidad, Paidós, Barcelona 2007.

Cortina, A., Neuroética y neuropolítica. Sugerencias para la educación moral, Tecnos, Madrid 2011.

Coy, J. J. (Dir.), La Declaración de la independencia. La Declaración de Seneca Falls, Traducción de María Coy Girón, Universidad de León, León 1993.

CuRia, D. (2016), «La importancia de llamarse Paul», Página 12, [En línea] Disponible en http:/www.pagina12.com.ar/diario/suplementos/soy/1-4022-2015-06-06.html (Consultado el 19/06/2016).

De Beauvoir, S., El segundo sexo, Ediciones DeBolsillo, Buenos Aires 2012 [Original: Le Deuxième Sexe, Gallimard, París 1949].

De Gouges, O., Vindicación de los Derechos de la Mujer, [Original: Déclaration des droits de la femme et de la citoyenne, 1789], [En línea] Disponible en http://clio.rediris.es/ n31/derechosmujer.pdf (Consultado el 19/06/2016).

Fausto-Sterling, A., Myths of Gender: Biological Theories about Women and Men, Basic Books, New York 1992.

FInE, C., Delusions of Gender: How Our Minds, Society and Neurosexism Create Difference, Icon Books, London 2010.

FIsher, M., «Não chore, pesquise! - Reflexões sobre sexo, gênero e ciência a partir do neurofeminismo» en: Anais da ReACT - V Reuniao de Antropologia da Ciência e da Tecnología, Vol.2, No2, 2015, 1-23.

Foucault, M., Tecnologías del yo y otros textos afines, Paidós, Barcelona 2008.

Friedan, B., La mística de la feminidad, Ediciones Cátedra, València 2009, [Original: The Feminine Mystique, 1963].

García-MarzÁ, D. y FeEnstra, R. A. (Eds.), Ética y neurociencias. La aportación a la política, la economía y la educación, Publicacions de la Universitat Jaume I, Castelló 2013.

Jordan-Young, R. y Rumiati, R., "Hardwire for Sexism? Approaches to Sex/Gender in Neuroscience», en: Bluhm, R., Jacobson, A. J. y Maibom, H. L. (Eds.), Neurofeminism. Issues at the intersection of feminist theory and cognitive science, Palgrave MacMillan, New York 2012, 105-120.

Kraus, C., "Critical Studies of the Sexed Brain: A Critique of What and for Whom?» en: Neuroethics, Vol. 5, N³, 2012, 247-259. 
Longino, H., «Essential Tensions-Phase Two: Feminist, Philosophical, and Social Studies of Science», en: Anthony, L. y Witt, C. (Coords.), A Mind of One's Own: Feminist Essays on Reason \& Objectivity, Westview Press, Boulder 1993.

Pallarés-Domínguez, D. V., "Críticas y orientaciones para el estudio en Neuroética» en: RECERCA. Revista de pensament $i$ anàlisi, $\mathrm{N}^{\circ} 13,2013,85-102$.

Pallarés-Domínguez, D. V., «La neurociencia aplicada al estudio de género: ¿una nueva perspectiva?» en: Fòrum de Recerca, Nº16, 2011, 17-35.

Preciado, P. B., Testo yonki, Espasa Calpe, Madrid 2008.

Preciado, P. B., Manifiesto contra-sexual, Editorial Opera Prima, Madrid 2002.

Reverter-Bañón, S., "Reflexión crítica frente al neurosexismo» en: Revista Pensamiento, Vol. $72 n^{\circ}$ X, 2016, pp. 959-979.

Reverter-Bañón, S., "La perspectiva de género en la filosofía» en: Feminismo/s, $\mathrm{N}^{\circ} 1$, 2003, 33-50.

RodrígueZ-Sierra, O. E., "A representação binária do cérebro “feminino" e "masculino" na ciência e nos meios de comunicação" en: Revista da Biologia, Vol.15 Nº1, 2016, 56-64.

SalLES, A., "On the Normative Implications of Social Neuroscience» en: RECERCA. Revista de pensament $i$ anàlisi, $\mathrm{N}^{\circ} 13,2013,29-42$.

Sсотт, J., «El género: una categoría útil para el análisis histórico» en: Lamas, Marta (Comp.), El género: la construcción cultural de la diferencia sexual, PUEG, México 1996, 265-302.

Wollstonecraft, M. (2000): Vindicación de los Derechos de la Mujer, Ediciones Cátedra, Valencia, [Original: A Vindication of the Right of Woman, 1792].

Universitat Jaume I

Maria Medina-Vicent

medinam@uji.es

[Artículo aprobado para publicación en octubre de 2016]. 